\title{
A Formative eAssessment Co-Design Case Study
}

\author{
D.A. Bacigalupo ${ }^{1}$, W.I. Warburton ${ }^{1}$, E.A. Draffan ${ }^{2}$, P. Zhang $^{2}$, L. Gilbert ${ }^{2}$, G.B. Wills ${ }^{2}$ \\ ${ }^{1}$ iSolutions, ${ }^{2}$ Learning Societies Lab School of Electronics and Computer Science \\ University of Southampton, SO17 1BJ, UK \\ db1f08@ecs.soton.ac.uk
}

\begin{abstract}
Formative eAssessment can be very helpful in providing high quality higher education assignments. However, there are obstacles restricting the uptake of formative eAssessment in higher education including both cultural and technical issues. When a university is encouraging the uptake of formative eAssessment internally it is useful to have case studies from academic schools detailing how academics enthusiastic about formative eAssessment have used it in their modules. It is particularly helpful if these case studies document: $i$.) the principle obstacles that these champions had to deal with; ii.) a cooperative-design process through which these obstacles have been dealt with by the champions (with assistance from e.g. learning technologists); and iii.) an evaluation of the effectiveness of the resulting formative eAssessments. However there is a shortage of such real-world long-term case studies. This paper helps fill this gap in the literature by describing the case of a Modern Languages module within a Russell Group university (Southampton). The formative eAssessment solution resulting from the case study utilises our QTI, mobile QTI, accessibility, and web 2.0 tools and can be positioned at the cutting edge of formative eAssessment practice. We have evaluated this with undergraduate student volunteers from Spanish modules and received positive feedback.
\end{abstract}

Keywords - formative eAssessment, mobile eAssessment, modern languages, web 2.0, accessibility, repository

\section{INTRODUCTION}

There is significant evidence that formative eAssessment can be helpful in providing high-quality higher education assignments [1,2]. In addition to doing eAssessments at a desk students can also use mobile devices, giving the student more flexibility in where they do the eAssessment and on what device [3]. However, there are obstacles restricting the uptake of formative eAssessment in higher education including both cultural and technical issues [4]. Models and studies of these obstacles include general technology uptake studies [5, 6, 7] and higher educationspecific studies $[4,8,9,10]$.

It is useful to have case studies from a range of academic schools detailing how academics enthusiastic about formative eAssessment (known as "champions") have applied formative eAssessment (or attempted to apply it) to their modules. It is particularly helpful if three key case study requirements are met. These involve documenting: $i$.) the principle obstacles that these champions had to deal with; ii.) a cooperative-design process through which these obstacles were dealt with by champions with assistance from e.g. learning technologists; and iii.) an evaluation of the effectiveness of the resulting formative eAssessments.
Examples of previous case studies include [11, 12, 13]. However, there is a shortage of real-world long-term case studies that meet the three key case study requirements as listed above. This paper helps fill this gap in the literature by describing a case study that meets these three requirements. This case study is particularly relevant to the understanding of e-Assessment uptake because it is: $i$.) lead by a non-IT specialist academic who is making significant use of formative eAssessment whilst not being involved in e-Assessment research outside of this case study; ii.) because it is conducted within a university having a strong interest in the uptake of formative eAssessment at an institutional level; and because iii.) formative eAssessment has been used on the module for $\sim 10$ years (primarily as a tool for motivated students to use independently).

The contributions of this paper are: i.) the co-design process and discussion of the obstacles; ii.) the subsequent implementation using our QTI, mobile QTI, accessibility, and web 2.0 tools; and iii.) the evaluation of the resulting eAssessments with undergraduate students from Spanish modules. This paper is structured into the following sections: (II) the case study and obstacles; (III) the codesign; (IV) the implementation; and (V) the evaluation.

\section{The CASE StUdy AND OBSTACLES}

A University of Southampton School of Humanities Spanish module for final year undergraduates is used as a case study. Prior to the work in this paper the optional independent study web-based formative eAssessments were implemented using Hot Potatoes. They required the students to first play an audio/video media file (in Spanish). Categories of eAssesment that could be automatically marked included: i.) assessing understanding of the media file (typically multiple choice questions); ii.) transcribing the media file using closed procedure (fill in the gap) questions; and iii.) a vocabulary test (also multiple choice). A small number of eAssessments were available each week for two semesters.

Obstacles to the enhancement of this process based on interviews with the course lecturer included the following cultural/institutional issues: (1) lack of confidence in university eAssessment support for Hot Potatoes (not the university recommended system); (2) difficulty motivating students to take full advantage of eAssessments; (3) the need to improve eAssessment accessibility and usability, especially for students with learning difficulties; and (4) writing high quality questions being difficult due to lack of examples of good and bad subject specific eAssessment questions, and associated guidance. Technical obstacles 
included: (5) interoperability of different eAssessment software; (6) how to choose the infrastructure technologies to use; (7) the need to increase usability of eAssessment systems for the lecturer; and (8) how to present results from a significant number of eAssessments in a form that allows them to be understood and made use of in a short period of time. These obstacles and the strategies we used to work through them, are discussed in sections III to V.

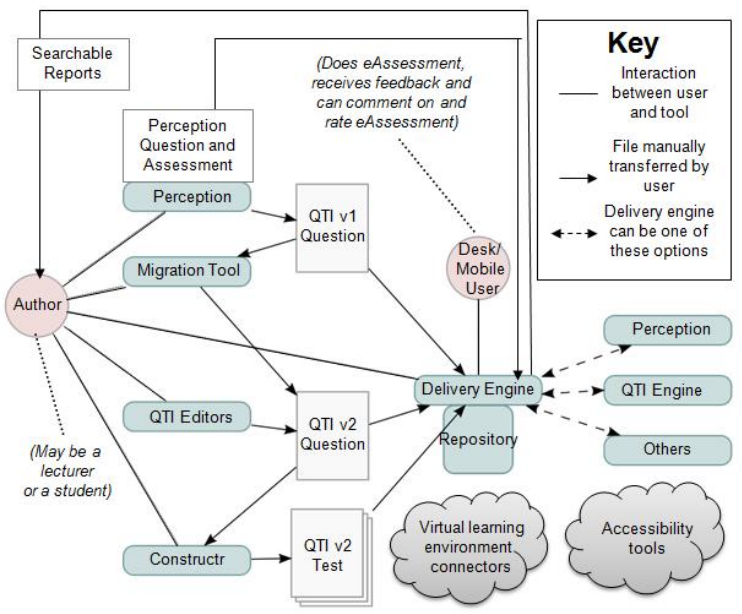

Figure 1: The design of our solution for formative eAssessment.

\section{THE Co-DesigN}

Our co-design process [14] involved developing personas, scenarios, design documentation etc. Figure 1 gives an overview of the design. We manually converted the eAssessments into QuestionMark Perception format so as to be fully supported by the University (see obstacle 1). Actions to help address obstacle 5 included exporting (using the JISC migration tool) the eAssessments into the standard QTI v2 format. Addressing obstacle 6 thus involved choosing the standard university infrastructure. QTI v2 questions can also be edited directly using a QTI editor. Our Constructr tool can be used to create a QTI test from QTI questions. The Perception/QTI eAssessment is done by a student using a delivery engine. The eAssessment is typically stored in a repository and accessed via a VLE.

These tools (with appropriate support) help address obstacle 7. To help address obstacle 3 we have: $i$.) followed human computer interaction advice where possible and advice from our JISC LexDis project [15]; ii.) conducted a usability evaluation - see section V; and iii.) incorporated web accessibility tools via our JISC TechDis ToolBar [16]. By tackling obstacle 3 and moving to the university eAssessment system we have helped address obstacle 2. In addition, we classify all eAssessment marks via a traffic light system so the lecturer can work with the students towards "green" results. This approach, combined with categorizing each eAssessment by subject, difficulty and question type helped to address obstacle 8 . In addition, in Web 2.0 fashion we allow students to provide feedback on eAssessments. To help address obstacle 4 we have extended the University's eAssessment training programme to include a workshop (and booklet) on pedagogically based eAssessment design.

\section{THE IMPLEMENTATION}

We have implemented a prototype eAssessment solution using the design from section III. New software includes: $i$.) a version of our QTI Engine delivery engine so eAssessments can be done from a (currently Android only) mobile device without a network connection; ii.) the Eqiat QTI and mobile QTI editors; iii.) our QTIbox Edshare repository plugin; and iv.) VLE connectors. As recommended in [17] feedback is specific (both perquestion and per-eAssessment), appears immediately, and is contingent on each student's answers. The content is provided by the lecturer, although we have other "Web 2.0" case studies where it is provided by the students. For further details and source code please see the EASiHE project website e.g. $[3,18]$.

\section{Evaluation}

The evaluation of the eAssessments was guided by [19] and took place at level 1 (the reaction level); that is, the reaction of the student. Each student evaluation (taking 11.5 hours) involved: $i$.) the interviewer explaining what is required; ii.) the student doing a pre-selected set of Perception eAssessments; and iii.) the students answering the evaluation questions (shown in table 1). The evaluation questions were decided upon after consultation with the module lecturer as well as learning technologists and the university CAA officer. Students on Spanish modules were invited to attend by the course lecturer, and a gift voucher was provided as inducement. All those responding to the course lecturer's email invitation who could attend the evaluation sessions were included.

TABLE I. THE EVALUATION QUESTIONS, ANSWERED ON A FIVE-POINT LIKERT SCALE

1. The exercises helped me learn

2. The exercises contributed to my general knowledge of what is going on in the Spanish speaking world

3. The exercises helped me learn new vocabulary

4. The exercises helped me improve my grammar

5. The system is easy to use

6. Compared to studying Spanish without any interactive exercises, I feel that the interactive video enhanced my learning

7. I would recommend that other modules or other languages courses provide some exercises like this

Five students were recruited to undertake the evaluation interviews. Three of these evaluations were conducted as described above. All these students answered "strongly agree" or "agree" to questions 1-5 and "strongly agree" to questions 6 and 7 - with the exception of one "disagree" response to question 6. All the students liked how the eAssessments helped motivate them in their independent learning. Dislikes included the material could be "more fun", and one student was "not a big fan of multiple choice". All students made comments implying that more 
care should be taken in classifying the difficulty of each eAssessment, as there are many factors (e.g. accents in video, student language background) that can affect this. We also asked two students to do a Perception eAssessment on a mobile device; both students found this straightforward and were positive about this experience. The final two interviews were about eAssessment accessibility. These highlighted technical requirements such as the importance of being able to automatically change fonts, colours and background (e.g. using our TechDis Toolbar). Also, cultural/institutional requirements were highlighted. For example, lecturers on other modules being more aware of accessibility issues and following the associated advice (e.g. from our LexDis project [15]) - such as using accessible page templates and keeping page layout and structure simple where possible. Overall, the student response to the eAssessments was positive, in particular in recommending that this kind of eAssessment be used on other modules.

The following is a summary of the internal EASiHE project case study evaluation [18]. We have taken steps to address obstacles 1 to 8 (see sections II and III) and learnt how to create a set of open source formative eAssessment tools (see section IV). A big challenge was how to manage risk when introducing new types of eAssessment onto a module, whilst keeping the lecturer's time commitment reasonable. We had 8 hours of co-design meetings with the lecturer over 5 months, whereas the lecturer would have preferred fewer meetings. We recommend planning and preparing eAssessments the term before the module runs. It seems likely that the School will continue using formative eAssessments and there appears to be a strong possibility that their use will be extended into other curriculum areas. This is something we will monitor. At an institutional level, favourable feedback has led to the leader of a Universitywide Assessment Review panel seeking input from the EASiHE management team on a long-term basis.

\section{CONCLUSION}

We have described a case study detailing how an academic "champion", together with learning technologists and IT staff, has used formative eAssessment in an undergraduate module. We identified eight technical /cultural obstacles and followed a co-design process to deal with them. The resulting solution can be positioned at the cutting edge of formative eAssessment practice. We have evaluated this with student volunteers from the module and received positive feedback. Future work includes publishing the results of our second evaluation and working with the University on embedding and helping to increase uptake.

\section{ACKNOWLEDGMENT}

Study funded by the JISC-Southampton EASiHE project.

\section{REFERENCES}

[1] E. Heinrich, J. Milne, M. Moore, An Investigation into ETool Use for Formative Assignment Assessment - Status and
Recommendations. Educational Technology \& Society, 12(4): 176-192, 2009

[2] N. Pachler, H. Mellar, C. Daly, Y. Mor, D. Wiliam, Scoping a vision for formative e-assessment: a project report for JISC, JISC project report, 2009. Available at www.jisc.ac.uk

[3] P. Zhang, G.B. Wills, L. Gilbert, B. Warburton, D. Bacigalupo, IMS QTI Engine on Android to Support Offline Mobile Learning, International Conference on e-Commerce, e-Administration, e-Society, e-Education, and e-Technology, Macau, China, 2010. Available at: easihe.ecs.soton.ac.uk.

[4] W. Warburton, Towards a grounded theory of computerassisted assessment uptake in UK universities, University of Southampton PhD Thesis, 2006

[5] E.M. Rogers, Diffusion of Innovations (5th edn.), New York: Free Press, 2003

[6] K. Lewin, Group Decisions and Social Change. In Readings in Social Psychology, G. E. Swanson, T. M. Newcomb and E. L. Hartley (Eds.) New York: Holt, Rhinehart and Winston, 1958

[7] G.A. Moore, Crossing the chasm (2nd edn.), Oxford, Eng.: Capstone Publishing, 1999

[8] The Association for Learning Technology, The Future of Higher Education: The Association for Learning Technology's response to the White Paper, 2003. Available at: www.alt.ac.uk/docs/he_wp_20030429_final.doc

[9] E. Sommerlad, M. Pettigrew, C. Ramsden, E. Stern, Synthesis Of TLTP Annual Reports, Tavistock Institute, London, 1999

[10] W. Warburton, Quick Win or Slow Burn? Modelling UK HE CAA Uptake, Assessment \& Evaluation in Higher Education, 34(3): 257 - 272, Routledge Press, 2009

[11] N. Pachler, C. Dalya, Y. Mora, H. Mellar, Formative eassessment: Practitioner Cases, Computers \& Education, Elsevier, 2009. In Press.

[12] K. Shephard, B. Warburton, P. Maier, A. Warren, Development and evaluation of computer-assisted assessment in higher education in relation to BS7988, Assessment \& Evaluation in Higher Education, 31:5(583595), Routledge Press, 2006

[13] JISC Pattern Language Network, case studies wiki, available at: http://purl.org/planet/Cases/, 2010.

[14] D. Millard, Y. Howard, L. Gilbert, G. Wills, Co-design and Co-deployment Methodologies for Innovative m-Learning Systems, In Multiplatform E-Learning Systems and Technologies: Mobile Devices for Ubiquitous ICT-Based Education, IGI Global, 2009

[15] J. Seale, E.A. Draffan, M. Wald, Exploring disabled learners' experiences of e-learning: LEXDIS Project Report. University of Southampton. Available at: http://eprints.soton.ac.uk/64850/

[16] S. Skuse, E.A. Draffan, M. Wald, JISC Techdis Toolbar manual installation and user guide, available at: http://access.ecs.soton.ac.uk/ToolBar/instructions, 2010

[17] L. Gilbert, V. Gale, Principles Of E-Learning Systems Engineering, Chandos Publishing 2007

[18] D. Bacigalupo, B. Warburton, L. Gilbert, G. Wills, The EASiHE Project: Case Study Details, 2010. Available at: http://easihe.ecs.soton.ac.uk/.

[19] D.L. Kirkpatrick, Evaluating Training Programs: The Four Levels (2nd Edn.), Berrett-Koehler Press, 1998. 